\title{
Equipolar meromorphic functions sharing a set
}

\author{
Arindam Sarkar
}

\begin{abstract}
Two meromorphic functions $f$ and $g$ having the same set of poles are known as equipolar. In this paper we study some uniqueness results of equi-polar meromorphic functions sharing a finite set and improve some recent results of Bhoosnurmath-Dyavanal [4] and Banerjee-Mallick [3] by removing some unnecessary conditions on ramification indices as well as relaxing the condition on the nature of sharing of the value $\infty$ by $f$ and $g$ from counting multiplicity to ignoring multiplicity.
\end{abstract}

Mathematics Subject Classification (2010): 30D35.

Keywords: Meromorphic function, uniqueness, set sharing.

\section{Introduction, definitions and results}

Let $f$ and $g$ and be two non-constant meromorphic functions defined in the open complex plane $\mathbb{C}$. If for some $a \in \mathbb{C} \cup\{\infty\}, f$ and $g$ have the same set of $a$-points with the same multiplicities, we say that $f$ and $g$ share the value $a \mathrm{CM}$ (Counting Multiplicities) and if we do not consider the multiplicities, then $f$ and $g$ are said to share the value $a$ IM (Ignoring Multiplicities). We do not explain the standard notations and definitions of the value distribution theory as these are available in [7].

Let $a \in \mathbb{C} \cup\{\infty\}$. We denote by $N(r, a ; f \mid=1)$, the counting function of the zeros of $f-a$ of multiplicity one. We also denote by $N(r, a ; f \mid \geq l)$, the counting function of those $a$-points of $f$ whose multiplicities are $\geq l$. Similarly we denote by $\bar{N}(r, a ; f \mid \geq l)$ the reduced counting function of the $a$-points of $f$ of multiplicity $\geq l$. We put $N_{2}(r, a ; f)=\bar{N}(r, a ; f)+\bar{N}(r, a ; f \mid \geq 2)$. We put

$$
\begin{aligned}
& \Theta(a ; f)=1-\limsup _{r \rightarrow \infty} \frac{\bar{N}(r, a ; f)}{T(r, f)} ; \\
& \delta_{2}(a ; f)=1-\limsup _{r \rightarrow \infty} \frac{N_{2}(r, a ; f)}{T(r, f)}
\end{aligned}
$$


and

$$
\delta_{(2}(a ; f)=1-\limsup _{r \rightarrow \infty} \frac{N(r, a ; f \mid \geq 2)}{T(r, f)} .
$$

Let $S$ be a set of distinct elements of $\mathbb{C} \cup\{\infty\}$ and

$$
E_{f}(S)=\bigcup_{a \in S}\{(z, p) \in \mathbb{C} \times \mathbb{N}: z \text { is an } a \text {-point of } f \text { of multiplicity } p\},
$$

and

$$
\bar{E}_{f}(S)=\bigcup_{a \in S}\{(z, 1) \in \mathbb{C} \times \mathbb{N}: z \text { is an } a \text {-point of } f\} .
$$

If $E_{f}(S)=E_{g}(S)$, we say that $f$ and $g$ share the set $S$ CM (Counting Multiplicity). On the other hand if $\bar{E}_{f}(S)=\bar{E}_{g}(S)$, we say that $f$ and $g$ share the set $S$ IM (Ignoring Multiplicity).

It will be convenient to denote by $E$, any subset of nonnegative real numbers of finite measure not necessary the same in each of its occurrence.

In 1976, Gross [6] considered the uniqueness problem of meromorphic functions when the functions under consideration share sets instead of values. In this direction Gross raised the following question:

Can one find finite sets $S_{j}, j=1,2$ such that any two non-constant entire functions $f$ and $g$ satisfying $E_{f}\left(S_{j}\right)=E_{g}\left(S_{j}\right)$ for $j=1,2$ must be identical ? results.

To answer the Question of Gross [6], in 1995, Yi [13] obtained the following

Theorem A. [13] Let $S=\left\{z: z^{n}+a z^{n-m}+b=0\right\}$, where $n$ and $m$ are two positive integers such that $m \geq 2, n \geq 2 m+7$, with $m$ and $n$ having no common factor, $a$ and $b$ be two nonzero constants such that $z^{n}+a z^{n-m}+b=0$ has no multiple root. If $f$ and $g$ be two non-constant meromorphic functions having no simple poles such that $E_{f}(S)=E_{g}(S)$ and $E_{f}(\{\infty\})=E_{g}(\{\infty\})$, then $f \equiv g$.

Theorem B. [13] Let $S=\left\{z: z^{n}+a z^{n-1}+b=0\right\}$, where $n(\geq 9)$ be an integer and $a$ and $b$ be two nonzero constants such that $z^{n}+a z^{n-1}+b=0$ has no multiple root. If $f$ and $g$ be two non-constant meromorphic functions such that $E_{f}(S)=E_{g}(S)$ and $E_{f}(\{\infty\})=E_{g}(\{\infty\})$, then either $f \equiv g$ or

$$
f \equiv \frac{-a h\left(h^{n-1}-1\right)}{h^{n}-1} \quad \text { and } \quad g \equiv \frac{-a\left(h^{n-1}-1\right)}{h^{n}-1}
$$

where $h$ is a non-constant meromorphic function.

Lahiri [8], in an attempt to investigate under which situation, $f \equiv g$, proved the following result.

Theorem C. [8] Let $S$ be defined as in Theorem $B$ and $n(\geq 8)$ be an integer. If $f$ and $g$ be two non-constant meromorphic functions having no simple poles such that $E_{f}(S)=E_{g}(S)$ and $E_{f}(\{\infty\})=E_{g}(\{\infty\})$, then $f \equiv g$.

Fang and Lahiri [5], improved Theorem $\mathrm{C}$ by reducing the cardinality of the same range set in the following result. 
Theorem D. [5] Let $S=\left\{z: z^{n}+a z^{n-1}+b=0\right\}$, where $n(\geq 7)$ be an integer and $a$ and $b$ be two nonzero constants such that $z^{n}+a z^{n-1}+b=0$ has no multiple root. If $f$ and $g$ be two non-constant meromorphic functions having no simple poles such that $E_{f}(S)=E_{g}(S)$ and $E_{f}(\{\infty\})=E_{g}(\{\infty\})$, then $f \equiv g$.

Below we give the definition of weighted sharing which will be required in the sequel.

Definition 1.1. $[9,10]$ Let $k$ be a nonnegative integer or infinity. For $a \in \mathbb{C} \cup\{\infty\}$, we denote by $E_{k}(a ; f)$ the set of all a-points of $f$ where an a-point of multiplicity $m$ is counted $m$ times if $m \leq k$ and $k+1$ times if $m>k$. If $E_{k}(a ; f)=E_{k}(a ; g)$, we say that $f$ and $g$ share the value a with weight $k$.

The definition implies that if $f, g$ share a value $a$ with weight $k$, then $z_{0}$ is a zero of $f-a$ with multiplicity $m(\leq k)$ if and only if it is a zero of $g-a$ with multiplicity $m(\leq k)$ and $z_{0}$ is a zero of $f-a$ of multiplicity $m(>k)$ if and only if it is a zero of $g-a$ with multiplicity $n(>k)$ where $m$ is not necessarily equal to $n$.

We write $f, g$ share $(a, k)$ to mean $f, g$ share the value $a$ with weight $k$. Clearly if $f, g$ share $(a, k)$ then $f, g$ share $(a, p)$ for all integers $p, 0 \leq p<k$. Also we note that $f, g$ share a value $a$ IM or CM if and only if $f, g$ share $(a, 0)$ or $(a, \infty)$ respectively.

Definition 1.2. [10] Let $S \subset \mathbb{C} \cup\{\infty\}$ and $k$ be a positive integer or $\infty$. We denote by $E_{f}(S, k)$ the set $\bigcup_{a \in S} E_{k}(a ; f)$.

Recently Bhoosnurmath-Dyavanal [4] proved the following result as an improvement of the above results by reducing the cardinality of the shared set $S$ as well as weakening the condition on ramification indices.

Theorem E. [4] Let $S=\left\{z: z^{n}+a z^{n-1}+b=0\right\}$, where $n(\geq 5)$ be an integer and $a$ and $b$ be two nonzero constants such that $z^{n}+a z^{n-1}+b=0$ has no multiple root. If $f$ and $g$ be two non-constant meromorphic functions such that $E_{f}(S, \infty)=E_{g}(S, \infty)$ and $E_{f}(\{\infty\}, \infty)=E_{g}(\{\infty\}, \infty)$. Also $N(r, 0 ; f \mid=1)=S(r, f)$ and $N(r, 0 ; g \mid=1)=$ $S(r, g)$ and $\Theta(\infty ; f)>\frac{2}{n-1}$ and $\Theta(\infty ; g)>\frac{2}{n-1}$, then $f \equiv g$. follows.

With the aid of weighted sharing Banerjee-Mallick [3] improved Theorem E as

Theorem F. [3] Let $S=\left\{z: z^{n}+a z^{n-1}+b=0\right\}$, where $n(\geq 5)$ be an integer and $a$ and $b$ be two nonzero constants such that $z^{n}+a z^{n-1}+b=0$ has no multiple root. If $f$ and $g$ be two non-constant meromorphic functions satisfying $E_{f}(S, m)=E_{g}(S, m)$ and $E_{f}(\{\infty\}, \infty)=E_{g}(\{\infty\}, \infty)$. Also $N(r, 0 ; f \mid=1)=S(r, f)$ and $N(r, 0 ; g \mid=1)=$ $S(r, g)$ and $\Theta_{f}+\Theta_{g}>\frac{4}{n-1}$. If

(i) $m \geq 2$ and $n \geq 5$;

(ii) or $m=1$ and $n \geq 6$;

(iii) or $m=0$ and $n \geq 10$,

then $f \equiv g$, where $\Theta_{f}=\delta_{(2}(0 ; f)+\Theta(\infty ; f)+\Theta\left(-a \frac{n-1}{n} ; f\right)$ and $\Theta_{g}$ is defined similarly. 
In this paper we give two-fold improvements to Theorem $\mathrm{F}$ as follows. Firstly we show that we can reach the conclusion of Theorem $\mathrm{F}$ without assuming the condition

$$
\Theta_{f}+\Theta_{g}>\frac{4}{n-1} \text {. }
$$

Secondly, we prove our theorem merely assuming that $f$ and $g$ share the value $\infty$ with weight 0 . That is we reduce the CM sharing of $\infty$ by $f$ and $g$ to IM sharing. We also show that the cardinality of the shared set $S$ can be reduced to 9 from 10 when $m=0$. We state below our theorem.

Theorem 1.1. Let $S=\left\{z: z^{n}+a z^{n-1}+b=0\right\}$, where $n(\geq 5)$ be an integer and $a$ and $b$ be two nonzero constants such that $z^{n}+a z^{n-1}+b=0$ has no multiple root. Let $f$ and $g$ be two non-constant meromorphic functions satisfying $E_{f}(S, m)=E_{g}(S, m)$, $E_{f}(\{\infty\}, 0)=E_{g}(\{\infty\}, 0)$ and $N(r, 0 ; f \mid=1)=S(r, f)$ and $N(r, 0 ; g \mid=1)=S(r, g)$. Then, $f \equiv g$, if any one of the following holds.

(i) $m=2, n \geq 5$;

(ii) $m=1, n \geq 6$;

(iii) $m=0, n \geq 9$.

Definition 1.3. [10] Let $f$ and $g$ be two non-constant meromorphic functions such that $f$ and $g$ share $(a, 0)$ for $a \in \mathbb{C} \cup\{\infty\}$. Let $z_{0}$ be an a-point of $f$ with multiplicity $p$, and an a-point of $g$ of multiplicity $q$. We denote by $\bar{N}_{L}(r, a ; f)\left(\bar{N}_{L}(r, a ; g)\right)$ the reduced counting function of those a-points of $f$ and $g$ where $p>q(q>p)$. We denote by $\bar{N}_{*}(r, a ; f, g)$ the reduced counting function of those a-points of $f$ whose multiplicities differ from the corresponding a-points of $g$. Clearly $\bar{N}_{*}(r, a ; f, g)=\bar{N}_{*}(r, a ; g, f)$ and $\bar{N}_{*}(r, a ; f, g)=\bar{N}_{L}(r, a ; f)+\bar{N}_{L}(r, a ; g)$. We also denote by $N_{E}^{1)}(r, a ; f)$ the counting function of those a-points of $f$ and $g$ where $p=q=1$. similarly we denote by $\bar{N}_{E}^{(2}(r, a ; f)$, the reduced counting function of those a-points of $f$ such that $p=q \geq 2$.

\section{Lemmas}

In this section we present some lemmas which will be required to establish our results. Let $f$ and $g$ be two nonconstant meromorphic functions and we define

$$
F=\frac{f^{n-1}(f+a)}{-b}, \quad G=\frac{g^{n-1}(g+a)}{-b} .
$$

In the lemmas several times we use the function $H$ defined by

$$
H=\frac{F^{\prime \prime}}{F^{\prime}}-\frac{2 F^{\prime}}{F-1}-\frac{G^{\prime \prime}}{G^{\prime}}+\frac{2 G^{\prime}}{G-1} .
$$

Lemma 2.1. [12] Let $f$ be a non-constant meromorphic function and let

$$
R(f)=\frac{\sum_{k=0}^{n} a_{k} f^{k}}{\sum_{j=0}^{m} b_{j} f^{j}}
$$


be an irreducible rational function in $f$ with constant coefficients $\left\{a_{k}\right\}$ and $\left\{b_{j}\right\}$, where $a_{n} \neq 0, b_{m} \neq 0$. Then $T(r, R(f))=d T(r, f)+S(r, f)$, where $d=\max \{m, n\}$.

Lemma 2.2. [14] If $F, G$ be two non-constant meromorphic functions such that they share $(1,0)$ and $H \not \equiv 0$ then,

$N_{E}^{1)}(r, 1 ; F \mid=1)=N_{E}^{1)}(r, 1 ; G \mid=1) \leq N(r, H)+S(r, F)+S(r, G)$.

Lemma 2.3. [2] Let $f$ and $g$ be two nonconstant meromorphic functions sharing $(1, m)$, $0 \leq m<\infty$. Then

$\bar{N}(r, 1 ; f)+\bar{N}(r, 1 ; g)-N_{E}^{1)}(r, 1 ; f)+\left(m-\frac{1}{2}\right) \bar{N}_{*}(r, 1 ; f, g) \leq \frac{1}{2}[N(r, 1 ; f)+N(r, 1 ; g)]$.

Lemma 2.4. Let $H \not \equiv 0$ and $E_{f}(S, 0)=E_{g}(S, 0)$ and $E_{f}(\{\infty\}, 0)=E_{g}(\{\infty\}, 0)$. Then, if $F$ and $G$ be given by (2.1),

$$
\begin{aligned}
& N(r, H) \\
\leq & \bar{N}(r, 0 ; F \mid \geq 2)+\bar{N}(r, 0 ; G \mid \geq 2)+\bar{N}(r, c ; F \mid \geq 2)+\bar{N}(r, c ; G \mid \geq 2) \\
+ & \bar{N}_{*}(r, 1 ; F, G)+\bar{N}_{*}(r, \infty ; F, G)+\bar{N}_{0}\left(r, 0 ; F^{\prime}\right)+\bar{N}_{0}\left(r, 0 ; G^{\prime}\right)+S(r, F) \\
+ & S(r, G),
\end{aligned}
$$

for $c \in \mathbb{C} \backslash\{0,1\}$. Here, $\bar{N}_{0}\left(r, 0 ; F^{\prime}\right)$, denotes the reduced counting function of the zeros of $F^{\prime}$, which are not the zeros of $F(F-1)(F-c)$. Similarly we define $\bar{N}_{0}\left(r, 0 ; G^{\prime}\right)$.

Proof. From the definition of $H$, it follows that that the poles of $H$ occur at the

(i) multiple zeros of $F$ and $G$;

(ii) poles of $F$ and $G$ of different multiplicities;

(iii) 1-points of $F$ and $G$ of different multiplicities;

(iv) multiple $c$-points of $F$ and $G$;

(v) the zeros of $F^{\prime}$ which are not the zeros of $F(F-1)(F-c)$;

(vi) the zeros of $G^{\prime}$ which are not the zeros of $G(G-1)(G-c)$.

Since the poles of $H$ are all simple, the lemma follows easily.

Lemma 2.5. [11] If two non-constant meromorphic functions $f$ and $g$ share $(\infty, 0)$. Then $f^{n-1}(f+a) g^{n-1}(g+a) \not \equiv b^{2}$, for $n \geq 2$.

Lemma 2.6. Let $f$ and $g$ be two non-constant meromorphic functions such that $f^{n-1}(f+a) \equiv g^{n-1}(g+a)$, where $n \geq 5$ is an integer. If $N(r, 1 ; f \mid=1)=S(r, f)$ and $N(r, 1 ; g \mid=1)=S(r, g)$, then $f \equiv g$.

Proof. Let

$$
f^{n-1}(f+a) \equiv g^{n-1}(g+a) .
$$

Clearly (2.2) implies that $f$ and $g$ share $(\infty, \infty)$. Suppose $f \not \equiv g$. Let $y=\frac{g}{f}$. Then (2.2) implies that $y \not \equiv 1, y^{n-1} \not \equiv 1, y^{n} \not \equiv 1$ and

$$
\begin{aligned}
& f \equiv-a \frac{1-y^{n-1}}{1-y^{n}} \\
\equiv & a\left(\frac{y^{n-1}}{1+y+y^{2}+\ldots+y^{n-1}}-1\right) \\
= & -a \frac{1+y+y^{2}+\ldots+y^{n-2}}{1+y+y^{2}+\ldots+y^{n-1}} .
\end{aligned}
$$


Case 1. Let $y=\frac{g}{f}=$ constant, then it follows from (2.3) that $f$ is constant, which is impossible.

Case 2. Let $y=\frac{g}{f}$ be non-constant.

Using Lemma 2.1, we note from $(2.2), T(r, f)=T(r, g)+O(1)$ and hence $S(r, f)=S(r, g)=S(r)$, say.

Let $z_{0}$ be a zero of $f+a$. Then in view of $(2.2), z_{0}$ must be a zero of either $g+a$ or $g$. If possible suppose that $z_{0}$ is a zero of $g+a$. Then $y\left(z_{0}\right)=1$ and from (2.3) we obtain $f\left(z_{0}\right)=-a\left(\frac{n-1}{n}\right) \neq-a$, that is $f\left(z_{0}\right)+a=-a\left(\frac{n-1}{n}\right) \neq 0$ which is a contradiction to our assumption. Therefore $z_{0}$ must be a zero of $g$. Thus we have

$$
\{z: f(z)+a=0\} \subseteq\{z: g(z)=0\} .
$$

Suppose $z_{0}$ be a zero of $f+a$ of multiplicity $p$ and a zero of $g$ of multiplicity $q$. Then in view of $(2.2), p=(n-1) q$. Thus $p=n-1$, if $q=1$ or $p \geq 2(n-1)$, when $q \geq 2$. Thus the least multiplicity of a zero of $f+a$ is $n-1$ and $f+a$ has no zero of multiplicity $m$ such that $n-1<m<2(n-1)$.

We agree to denote by $\bar{N}\left(r, 0 ; f+a \mid g_{=1}=0\right)$, the reduced counting function of the zeros of $f+a$ which are the zeros of $g$ of multiplicity $=1$ and by $\bar{N}(r, 0 ; f+a \mid$ $g_{\geq 2}=0$ ), the reduced counting function of the zeros of $f+a$ which are the zeros of $g$ of multiplicity $\geq 2$. Also we denote by $\bar{N}(r, 0 ; f+a \mid g=0)$ the reduced counting function of the zeros of $f+a$, which are the zeros of $g$.

Now since $N(r, 0 ; g \mid=1)=S(r, g)$, we have from (2.4) and above analysis,

$$
\begin{aligned}
& \bar{N}(r, 0 ; f+a) \\
= & \bar{N}(r, 0 ; f+a \mid g=0) \\
= & \bar{N}(r, 0 ; f+a \mid g=1=0)+\bar{N}\left(r, 0 ; f+a \mid g_{\geq 2}=0\right) \\
= & S(r, g)+\bar{N}(r, 0 ; f+a \mid \geq 2(n-1)) \\
= & S(r, f)+\bar{N}(r, 0 ; f+a \mid \geq 2(n-1)) .
\end{aligned}
$$

Hence

$$
(2 n-2) \bar{N}(r, 0 ; f+a) \leq T(r, f)+S(r, f) .
$$

From (2.3) we observe that $T(r, f)=(n-1) T(r, y)+S(r, y)$. Also

$$
\begin{aligned}
& f+a \frac{n-1}{n} \\
= & -a \frac{1-y^{n-1}}{1-y^{n}}+a \frac{n-1}{n} \\
= & -a \frac{(n-1) y^{n}-n y^{n-1}+1}{n\left(1-y^{n}\right)} .
\end{aligned}
$$

If we put $p(y)=(n-1) y^{n}-n y^{n-1}+1$, then $p(0) \neq 0$ and $p^{\prime}(y)=n(n-1) y^{n-2}\{y-1\}$ and $p^{\prime \prime}(y)=n(n-1) y^{n-3}\{(n-3) y-n+2\}$. Thus $p(1)=p^{\prime}(1)=0$. Hence $p(y)=0$ has only one repeated root at $y=1$. 
Thus from (2.5) we obtain

$$
\sum_{i=1}^{n-1} \bar{N}\left(r, u_{i} ; y\right) \leq \bar{N}\left(r,-a \frac{n-1}{n} ; f\right),
$$

where $u_{i}, i=1, \ldots, n-1$ are the distinct zeros of $p(y)$.

Also from (2.3) we have

$$
\sum_{j=1}^{n-1} \bar{N}\left(r, v_{j} ; y\right) \leq \bar{N}(r, \infty ; f) \leq T(r, f) .
$$

Since by our assumption $N(r, 0 ; f \mid=1)=S(r, f)$, we have

$$
\bar{N}(r, 0 ; f)=N(r, 0 ; f \mid=1)+\bar{N}(r, 0 ; f \mid \geq 2) \leq S(r, f)+\frac{1}{2} T(r, f) .
$$

Thus we have

$$
\begin{aligned}
& \sum_{j=1}^{n-2} \bar{N}\left(r, w_{j} ; y\right)+\bar{N}(r, \infty ; y) \\
\leq & \bar{N}(r, 0 ; f)=N(r, 0 ; f \mid=1)+\bar{N}(r, 0 ; f \mid \geq 2) \leq \frac{1}{2} T(r, f)+S(r, f),
\end{aligned}
$$

where $v_{j} \mathrm{~s}, j=1,2, \ldots, n-1$ are the distinct roots of $1+y+y^{2}+\ldots+y^{n-1}=0$ and $w_{j} \mathrm{~s}, j=1,2, \ldots, n-2$ are the distinct roots of $1+y+y^{2}+\ldots+y^{n-2}=0$.

From (2.2) and (2.3) we note that the zeros of $y$ occur at those zeros of $g$ which are the zeros of $f+a$. Hence $\bar{N}(r, 0 ; y) \leq \bar{N}(r, 0 ; f+a)$.

Also we have obtained $(2 n-2) \bar{N}(r, 0 ; f+a) \leq T(r, f)+S(r, f)$. Thus, we obtain by the second main theorem,

$$
\begin{aligned}
& (3 n-4) T(r, y) \\
\leq & \sum_{j=1}^{n-1} \bar{N}\left(r, v_{j} ; y\right)+\sum_{j=1}^{n-2} \bar{N}\left(r, w_{j} ; y\right)+\sum_{i=1}^{n-1} \bar{N}\left(r, u_{i} ; y\right)+\bar{N}(r, 0 ; y) \\
+ & \bar{N}(r, \infty ; y)+S(r, y) \\
\leq & \bar{N}(r, \infty ; f)+\bar{N}(r, 0 ; f)+\bar{N}\left(r,-a \frac{n-1}{n} ; f\right)+\bar{N}(r, 0 ; f+a) \\
+ & S(r, f) \\
\leq & \left\{1+\frac{1}{2}+1+\frac{1}{2 n-2}\right\} T(r, f)+S(r, f) \\
\leq & \left(\frac{5}{2}+\frac{1}{2 n-2}\right)(n-1) T(r, y)+S(r, y),
\end{aligned}
$$

which leads to a contradiction for $n \geq 5$. This completes the proof of the Lemma.

Lemma 2.7. Let $S=\left\{z: z^{n}+a z^{n-1}+b=0\right\}$, where $n(\geq 4)$ be an integer and $a$ and $b$ be two nonzero constants such that $z^{n}+a z^{n-1}+b=0$ has no multiple root. If $F$ and $G$ are given by (2.1), then there exists an $\alpha \in \mathbb{C} \backslash\{0, a, b\}$, satisfying $N_{2}(r, \alpha ; F) \leq(n-1) T(r, f)+S(r, f), N_{2}(r, \alpha ; G) \leq(n-1) T(r, g)+S(r, g)$, where 
$|\alpha|=\frac{(n-1)^{n-1}}{n^{n}} \cdot \frac{|a|^{n}}{|b|}, \arg \alpha=\arg \left(\frac{a^{n}}{b}\right)$ or $\arg \alpha=\arg \left(-\frac{a^{n}}{b}\right)$, according as $n$ is even or odd. Here $\arg z$ denotes the principal argument of $z$ for any $z \in \mathbb{C} \backslash\{0\}$.

Proof. Let $p(z)=z^{n}+a z^{n-1}+b$. Then $p^{\prime}(z)=z^{n-2}\{n z+a(n-1)\}$. Thus $p^{\prime}(z)=0$ has roots at $z=0$ and at $z=-\frac{a(n-1)}{n}$. Thus $p(z)=0$ will have a repeated root at $-\frac{a(n-1)}{n}$ provided $p\left(-\frac{a(n-1)}{n}\right)=0$ and this yields $b=(-1)^{n}\left(\frac{a}{n}\right)^{n}(n-1)^{n-1}$. Note that $p^{\prime \prime}\left(-\frac{a(n-1)}{n}\right) \neq 0$.

Thus $p(z)=0$ has a repeated root at $-\frac{a(n-1)}{n}$ and hence only $n-1$ distinct roots provided $b=(-1)^{n}\left(\frac{a}{n}\right)^{n}(n-1)^{n-1}$.

Let $\alpha$ be a nonzero complex number. Then

$$
F-\alpha=\frac{f^{n-1}(f+a)}{-b}-\alpha=\frac{f^{n}+a f^{n-1}+\alpha b}{-b} .
$$

We choose $\alpha$ in such a manner that the equation $z^{n}+a z^{n-1}+\alpha b=0$ has repeated roots. It is clear from the above discussion that in this case we must have

$$
\alpha b=(-1)^{n}\left(\frac{a}{n}\right)^{n}(n-1)^{n-1} .
$$

This implies $|\alpha|=\frac{(n-1)^{n-1}}{n^{n}} \cdot \frac{|a|^{n}}{|b|}, \arg \alpha=\arg \left(\frac{a^{n}}{b}\right)$ or $\arg \alpha=\arg \left(-\frac{a^{n}}{b}\right)$, according as $n$ is even or odd. If $w_{1}, w_{2}, \ldots, w_{n-1}$, be the distinct roots of $z^{n}+a z^{n-1}+\alpha b=0$, then we have

$$
\begin{aligned}
& N_{2}(r, \alpha ; F) \\
= & \bar{N}(r, \alpha ; F)+\bar{N}(r, \alpha ; F \mid \geq 2) \\
\leq & \sum_{i=1}^{n-1} \bar{N}\left(r, w_{i} ; f\right)+\sum_{i=1}^{n-1} \bar{N}\left(r, w_{i} ; f \mid \geq 2\right)+S(r, f) \\
= & \sum_{i=1}^{n-1}\left\{\bar{N}\left(r, w_{i} ; f\right)+\bar{N}\left(r, w_{i} ; f \mid \geq 2\right)\right\}+S(r, f) \\
= & \sum_{i=1}^{n-1} N_{2}\left(r, w_{i} ; f\right)+S(r, f) \\
\leq & (n-1) T(r, f)+S(r, f) .
\end{aligned}
$$

This completes the proof.

Lemma 2.8. Let $F, G$ be given by (2.1) and $V=\left(\frac{F^{\prime}}{F-1}-\frac{F^{\prime}}{F}\right)-\left(\frac{G^{\prime}}{G-1}-\frac{G^{\prime}}{G}\right) \not \equiv 0$. If $\bar{N}(r, 0 ; f \mid=1)=S(r, f)$ and $\bar{N}(r, 0 ; g \mid=1)=S(r, g)$ and $f, g$ share $(\infty, 0) ; F, G$, share $(1,0)$, then $\{n-1\} \bar{N}(r, \infty ; f) \leq\left\{\frac{1}{2}+1\right\}\{T(r, f)+T(r, g)\}+\bar{N}_{*}(r, 1 ; F, G)+S(r, f)+S(r, g)$. 
Proof. Let $z_{0}$ be a pole of $f$ and $g$ of respective multiplicities $p$ and $q$. Then from (2.1), around $z_{0}$, we have

$$
F=\frac{A(z)}{\left(z-z_{0}\right)^{n p}}, \quad G=\frac{B(z)}{\left(z-z_{0}\right)^{n q}} .
$$

Where $A(z)$ and $B(z)$ are analytic at $z_{0}$, and $A\left(z_{0}\right) \neq 0, B\left(z_{0}\right) \neq 0$.

Thus

$$
\frac{F^{\prime}}{F-1}=\frac{A^{\prime}}{A-\left(z-z_{0}\right)^{n p}}-\frac{n p A}{\left(z-z_{0}\right)\left[A-\left(z-z_{0}\right)^{n p}\right]}
$$

and

$$
\frac{F^{\prime}}{F}=\frac{A^{\prime}}{A}-\frac{n p}{z-z_{0}}
$$

Therefore a simple calculation yields,

$$
\begin{aligned}
\frac{F^{\prime}}{F-1}-\frac{F^{\prime}}{F} & =\left(z-z_{0}\right)^{n p-1}\left\{\frac{A^{\prime}}{A} \cdot \frac{z-z_{0}}{A-\left(z-z_{0}\right)^{n p}}-\frac{n p}{A-\left(z-z_{0}\right)^{n p}}\right\} \\
& =\left(z-z_{0}\right)^{n p-1} \phi(z),
\end{aligned}
$$

say, where $\phi(z)$ is analytic at $z_{0}$ and $\phi\left(z_{0}\right) \neq 0$. Similarly we obtain,

$$
\begin{aligned}
\frac{G^{\prime}}{G-1}-\frac{G^{\prime}}{G} & =\left(z-z_{0}\right)^{n q-1}\left\{\frac{B^{\prime}}{B} \cdot \frac{z-z_{0}}{B-\left(z-z_{0}\right)^{n q}}-\frac{n q}{B-\left(z-z_{0}\right)^{n q}}\right\} \\
& =\left(z-z_{0}\right)^{n q-1} \psi(z),
\end{aligned}
$$

say, where $\psi(z)$ is analytic at $z_{0}$ and $\psi\left(z_{0}\right) \neq 0$. Therefore, around $z_{0}$,

$$
V=\left(z-z_{0}\right)^{n p-1} \phi(z)-\left(z-z_{0}\right)^{n q-1} \psi(z) .
$$

Thus $V$ has a zero at $z_{0}$, of order at least $n-1$.

We note by Millux's theorem

$$
\begin{aligned}
& m(r, V) \\
= & m\left(r,\left(\frac{F^{\prime}}{F-1}-\frac{F^{\prime}}{F}\right)-\left(\frac{G^{\prime}}{G-1}-\frac{G^{\prime}}{G}\right)\right) \\
\leq & m\left(r, \frac{F^{\prime}}{F-1}\right)+m\left(r, \frac{G^{\prime}}{G-1}\right)+m\left(r, \frac{F^{\prime}}{F}\right)+m\left(r, \frac{G^{\prime}}{G}\right) \\
= & S(r, F)+S(r, G)=S(r, f)+S(r, g) .
\end{aligned}
$$

Hence from above analysis and by the first fundamental theorem, we have

$$
\begin{aligned}
& \{n-1\} \bar{N}(r, \infty ; f) \\
\leq & N(r, 0 ; V) \\
\leq & T(r, V)+O(1) \\
\leq & N(r, \infty ; V)+S(r, f)+S(r, g) \\
\leq & \bar{N}(r, 0 ; f)+\bar{N}(r, 0 ; g)+\bar{N}(r, 0 ; f+a)+\bar{N}(r, 0 ; g+a) \\
+ & \bar{N}_{*}(r, 1 ; F, G)+S(r, f)+S(r, g) .
\end{aligned}
$$


Now since $\bar{N}(r, 0 ; f \mid=1)=S(r, f)$ and $\bar{N}(r, 0 ; g \mid=1)=S(r, g)$, we have

$$
\bar{N}(r, 0 ; f) \leq \frac{1}{2} T(r, f)+S(r, f)
$$

and

$$
\bar{N}(r, 0 ; g) \leq \frac{1}{2} T(r, g)+S(r, g)
$$

Therefore from above, we have

$$
\begin{aligned}
& \{n-1\} \bar{N}(r, \infty ; f) \\
\leq & \left\{\frac{1}{2}+1\right\}\left\{T(r, f)+T(r, g\}+\bar{N}_{*}(r, 1 ; F, G)+S(r, f)+S(r, g) .\right.
\end{aligned}
$$

This completes the proof.

Lemma 2.9. [1] Let $F$ and $G$ be defined by (2.1) and $F$ and $G$ share $(1, m), 0 \leq m<$ $\infty$. Also let $w_{1}, \ldots, w_{n}$ be the distinct roots of the equation $z^{n}+a z^{n-1}+b=0$, where $b \neq(-1)^{n}\left(\frac{a}{n}\right)^{n}(n-1)^{n-1}, n \geq 3$. Then

$$
\bar{N}_{L}(r, 1 ; F) \leq \frac{1}{m+1}\{\bar{N}(r, 0 ; f)+\bar{N}(r, \infty ; f)\}-N_{\odot}\left(r, 0 ; f^{\prime}\right)+S(r, f),
$$

where $N_{\odot}\left(r, 0 ; f^{\prime}\right)=N\left(r, 0 ; f^{\prime} \mid f \neq 0, w_{1}, \ldots, w_{n}\right)$. Similar inequality holds for $\bar{N}_{L}(r, 1 ; G)$.

Lemma 2.10. Let $F$ and $G$ be defined by (2.1) and $F$ and $G$ share $(1, m), 0 \leq m<\infty$. Also let $\bar{N}(r, 0 ; f \mid=1)=S(r, f)$ and $\bar{N}(r, 0 ; g \mid=1)=S(r, g)$. Then

$$
\begin{aligned}
& \bar{N}_{*}(r, 1 ; F, G) \\
\leq & \frac{1}{m+1}\left\{\frac{1}{2}[T(r, f)+T(r, g)]+\bar{N}(r, \infty ; f)+\bar{N}(r, \infty ; g)\right\}+S(r, f)+S(r, g) .
\end{aligned}
$$

Proof. Since $\bar{N}_{*}(r, 1 ; F, G)=\bar{N}_{L}(r, 1 ; F)+\bar{N}_{L}(r, 1 ; G)$ and from the condition of the Lemma it follows that

$$
\bar{N}(r, 0 ; f) \leq \frac{1}{2} T(r, f)+S(r, f)
$$

and

$$
\bar{N}(r, 0 ; g) \leq \frac{1}{2} T(r, g)+S(r, g)
$$

the Lemma follows from Lemma 2.9.

Lemma 2.11. Let $F$ and $G$ be defined by (2.1) and $F$ and $G$ share $(1, m), 0 \leq m<\infty$. Also let $\bar{N}(r, 0 ; f \mid=1)=S(r, f)$ and $\bar{N}(r, 0 ; g \mid=1)=S(r, g)$ and $f$ and $g$ share $(\infty, 0)$. Then

$$
\begin{aligned}
& {\left[n-1-\frac{2}{m+1}\right] \bar{N}(r, \infty ; f) } \\
\leq & {\left[\frac{3}{2}+\frac{1}{2(m+1)}\right]\{T(r, f)+T(r, g)\} } \\
+ & S(r, f)+S(r, g) .
\end{aligned}
$$


Proof. From Lemmas 2.8 and 2.10, we have

$$
\begin{aligned}
& \{n-1\} \bar{N}(r, \infty ; f) \\
\leq & \left\{\frac{1}{2}+1+\frac{1}{2(m+1)}\right\}\left\{T(r, f)+T(r, g\}+\frac{2}{m+1} \bar{N}(r, \infty ; f)\right. \\
+\quad & S(r, f)+S(r, g) .
\end{aligned}
$$

The lemma follows easily from above.

\section{Proof of theorem}

Proof of Theorem 1.1. Case 1. $H \not \equiv 0$. By Lemma 2.1, we obtain from the definitions of $F$ and $G, T(r, F)=n T(r, f)+S(r, f), T(r, G)=n T(r, g)+S(r, g)$.

We denote by $N_{0}\left(r, 0 ; F^{\prime}\right)$, the counting function of the zeros of $F^{\prime}$ which are not the zeros of $F(F-1)(F-c)$, for some $c \in \mathbb{C} \backslash\{0,1\}$. Similarly we define $N_{0}\left(r, 0 ; G^{\prime}\right)$. Now applying the second main theorem to $F$ and $G$, we obtain for some $c \in \mathbb{C} \backslash\{0,1\}$,

$$
\begin{aligned}
& 2\{T(r, F)+T(r, G)\} \\
\leq & \bar{N}(r, 0 ; F)+\bar{N}(r, c ; F)+\bar{N}(r, 1 ; F)+\bar{N}(r, \infty ; F)+\bar{N}(r, 0 ; G)+\bar{N}(r, c ; G) \\
+ & \bar{N}(r, 1 ; G)+\bar{N}(r, \infty ; G)-N_{0}\left(r, 0 ; F^{\prime}\right)-N_{0}\left(r, 0 ; G^{\prime}\right)+S(r, f)+S(r, g),
\end{aligned}
$$

and hence

$$
\begin{aligned}
& 2 n\{T(r, f)+T(r, g)\} \\
\leq & \bar{N}(r, 0 ; F)+\bar{N}(r, c ; F)+\bar{N}(r, 1 ; F)+\bar{N}(r, \infty ; F)+\bar{N}(r, 0 ; G)+\bar{N}(r, c ; G) \\
+ & \bar{N}(r, 1 ; G)+\bar{N}(r, \infty ; G)-N_{0}\left(r, 0 ; F^{\prime}\right)-N_{0}\left(r, 0 ; G^{\prime}\right)+S(r, f)+S(r, g) .
\end{aligned}
$$

Using Lemma 2.2, Lemma 2.3 and 2.4 and 2.9 we have from above,

$$
\begin{aligned}
& 2 n\{T(r, f)+T(r, g)\} \\
\leq & N_{2}(r, 0 ; F)+N_{2}(r, c ; F)+3 \bar{N}(r, \infty ; f)+N_{2}(r, 0 ; G)+N_{2}(r, c ; G) \\
+ & \frac{n}{2}\{T(r, f)+T(r, g)\}+\left(\frac{3}{2}-m\right) \bar{N}_{*}(r, 1 ; F, G)+S(r, f)+S(r, g) \\
\leq & 2 \bar{N}(r, 0 ; f)+N_{2}(r, 0 ; f+a)+(n-1) T(r, f)+3 \bar{N}(r, \infty ; f) \\
+ & 2 \bar{N}(r, 0 ; g)+N_{2}(r, 0 ; g+a)+(n-1) T(r, g)+\frac{n}{2}\{T(r, f)+T(r, g)\} \\
+ & \left(\frac{3}{2}-m\right) \bar{N}_{*}(r, 1 ; F, G)+S(r, f)+S(r, g) .
\end{aligned}
$$


Subcase 1.1. $m=2$. We obtain from (3.1) using Lemma 2.8,

$$
\begin{aligned}
& \left(\frac{n}{2}-1\right)\{T(r, f)+T(r, g)\} \\
\leq & \bar{N}(r, \infty ; f)+\frac{2.3}{2(n-1)}\{T(r, f)+T(r, g)\}+\left(\frac{2}{n-1}-\frac{1}{2}\right) \bar{N}_{*}(r, 1 ; F, G) \\
+ & S(r, f)+S(r, g) . \\
\leq & \frac{1}{2}\{T(r, f)+T(r, g)\}+\frac{2.3}{2(n-1)}\{T(r, f)+T(r, g)\} \\
+ & \left(\frac{2}{n-1}-\frac{1}{2}\right) \bar{N}_{*}(r, 1 ; F, G)+S(r, f)+S(r, g) .
\end{aligned}
$$

But this leads to a contradiction for $n \geq 5$.

Subcase 1.2. $m=1$. Then proceeding as in Subcase 1.1, the Lemma 2.2 with $m=1$ and Lemma 2.3, yield the following.

$$
\begin{aligned}
& 2 n\{T(r, f)+T(r, g)\} \\
\leq & 2\left\{T(r, f)+T(r, g\}+(n-1)\left\{T(r, f)+T(r, g\}+\frac{n}{2}\{T(r, f)+T(r, g\}\right.\right. \\
+ & 3 \bar{N}(r, \infty ; f)+\frac{1}{2} \bar{N}_{*}(r, 1 ; F, G)+S(r, f)+S(r, g) .
\end{aligned}
$$

Using the Lemma 2.10 we obtain from above,

$$
\begin{aligned}
& \left(\frac{n}{2}-1\right)\{T(r, f)+T(r, g\} \\
\leq & 3 \bar{N}(r, \infty ; f)+\frac{1}{2} \cdot \frac{1}{1+1}\left[\frac{1}{2} T(r, f)+\frac{1}{2} T(r, g)+\bar{N}(r, \infty ; f)+\bar{N}(r, \infty ; g)\right] \\
+ & S(r, f)+S(r, g) \\
= & \left\{\frac{3}{2}+\frac{1}{4}\right\}[\bar{N}(r, \infty ; f)+\bar{N}(r, \infty ; g)]+\frac{1}{8}\{T(r, f)+T(r, g\} \\
+ & S(r, f)+S(r, g) \\
\leq & \left\{\frac{3}{2}+\frac{1}{4}+\frac{1}{8}\right\}\{T(r, f)+T(r, g\}+S(r, f)+S(r, g) .
\end{aligned}
$$

This leads to a contradiction for $n \geq 6$. 
Subcase 1.3. $m=0$. Proceeding as in Subcase 1.2., we obtain using Lemmas 2.10 and 2.11 with $m=0$,

$$
\begin{aligned}
& \left\{\frac{n}{2}-1\right\}\{T(r, f)+T(r, g\} \\
\leq & 3 \bar{N}(r, \infty ; f)+\frac{3}{2} \bar{N}_{*}(r, 1 ; F, G) \\
\leq & 3 \bar{N}(r, \infty ; f)+\frac{3}{2}\left\{\frac{1}{2} T(r, f)+\frac{1}{2} T(r, f)+\bar{N}(r, \infty ; f)+\bar{N}(r, \infty ; g)\right\} \\
+ & S(r, f)+S(r, g) \\
= & 6 \cdot \frac{2}{n-3}\{T(r, f)+T(r, g)\}+\frac{3}{4}\{T(r, f)+T(r, g)\}+S(r, f)+S(r, g) \\
= & \left(\frac{12}{n-3}+\frac{3}{4}\right)\{T(r, f)+T(r, g)\}+S(r, f)+S(r, g),
\end{aligned}
$$

this leads to a contradiction for $n \geq 9$.

Case 2. $H \equiv 0$. We have

$$
F \equiv \frac{A G+B}{C G+D}
$$

where $A D-B C \neq 0$. Clearly from above and the definitions of $F$ and $G$ we have $T(r, F)=T(r, G)+O(1)$ and $T(r, f)=T(r, g)+O(1)$.

Subcase 2.1. $A C \neq 0$. Since $f$ and $g$ share $\{\infty\}$, it follows from (3.2) that $\infty$ is an exceptional value of $f$ and $g$. So by the second main theorem we get,

$$
\begin{aligned}
& n T(r, f) \\
\leq & \bar{N}(r, 0 ; F)+\bar{N}(r, \infty ; F)+\bar{N}\left(r, \frac{A}{C} ; F\right)+S(r, f) \\
\leq & \bar{N}(r, 0 ; f)+\bar{N}(r, 0 ; f+a)+\bar{N}(r, \infty ; f)+\bar{N}(r, \infty ; g)+S(r, f) \\
\leq & 2 T(r, f)+S(r, f),
\end{aligned}
$$

which leads to a contradiction for $n \geq 5$.

Subcase 2.2. Let $A \neq 0$ and $C=0$. Then $F=\gamma G+\beta$, where $\gamma=\frac{A}{D} \neq 0$ and $\beta=\frac{B}{D}$. It is obvious that $F$ and $G$ cannot omit the value 1 . For if $F$ omits the value 1 , then $f$ (and $g$ as well) omits the distinct roots of the equation $z^{n}+a z^{n-1}+b=0$, which certainly leads to a contradiction for $n \geq 3$.

Thus $F$ and $G$ assume the value 1 and we have from above

$$
F=\gamma G+(1-\gamma)
$$

If $\gamma=1$ we have $F \equiv G$ and by Lemma 2.6 , we have $f \equiv g$.

So let $\gamma \neq 1$. Since $N(r, 0 ; f \mid=1)=S(r, f)$ and $N(r, 0 ; g \mid=1)=S(r, g)$, we have from (3.4) using the second main theorem,

$$
\begin{aligned}
& n T(r, f) \\
\leq & \bar{N}(r, 0 ; F)+\bar{N}(r, 1-\gamma ; F)+\bar{N}(r, \infty ; F)+S(r, f) \\
\leq & \frac{1}{2} T(r, f)+\bar{N}(r, 0 ; f+a)+\frac{1}{2} T(r, g)+\bar{N}(r, 0 ; g+a)+\bar{N}(r, \infty ; f)+S(r, f) \\
\leq & 4 T(r, f)+S(r, f) .
\end{aligned}
$$


This leads to a contradiction for $n \geq 5$.

Subcase 2.3. $A=0, C \neq 0$. Then clearly $B \neq 0$. Hence, $F \equiv \frac{1}{\zeta G+\eta}$. We can show as before that $F$ and $G$ cannot omit the value 1 and hence $F \equiv \frac{1}{\zeta G+1-\zeta}$. Let $\zeta=1$. Then $F G \equiv 1$. This is a contradiction by Lemma 2.5.

So $\zeta \neq 1$. Now since $f$ and $g$ share $\infty$, the relation $F \equiv \frac{1}{\zeta G+1-\zeta}$, at once implies $F$ cannot assume the values $\infty$ and 0 , and therefore $f$ cannot assume the values $\infty$, 0 and $-a$. This is impossible. This completes the proof of the theorem.

\section{References}

[1] Banerjee, A., Some uniqueness results on meromorphic functions sharing three sets, Ann. Polon. Math., 92(2007), 261-274.

[2] Banerjee, A., On the uniqueness of meromorphic functions sharing two sets, Comm. Math. Anal., 10(2010), no. 10, 1-10.

[3] Banerjee, A., Mallick, S., Uniqueness of meromorphic functions sharing two sets having deficient values, Matematychni Stud., 41(2014), no.2, 168-178.

[4] Bhoosnurmath, S.S., Dyavanal, R. , Uniqueness of meromorphic functions sharing sets, Bull. Math. Anal. Appl., 3(2011), no. 3, 200-208.

[5] Fang, M., Lahiri, I., Unique range set for certain meromorphic functions, Indian J. Math., 45(2003), no. 2, 141-150.

[6] Gross, F., Factorization of meromorphic functions and some open problems, Proc. Conf. Univ. Kentucky, Lexington, Ky (1976) Lecture Notes in Math., Springer (Berlin), 599(1977), 51-69.

[7] Hayman, W.K., Meromorphic Functions, Clarendon Press, Oxford 1964.

[8] Lahiri, I., The range set of meromorphic derivatives, Northeast Math. J., 14(1998), no. 3, 353-360.

[9] Lahiri, I., Weighted sharing and uniqueness of meromorphic functions, Nagoya Math. J., 161(2001), 193-206.

[10] Lahiri, I., Weighted value sharing and uniqueness of meromorphic functions, Complex Var. Theory Appl., 46(2001), 241-253.

[11] Lahiri, I., On a question of Hong Xun Yi, Arch Math. (Brno), 38(2002), 119-128.

[12] Mohon'ko, A.Z., On the Nevanlinna characteristics of some meromorphic functions, Theory of Funct. Funct. Anal. Appl., 14(1971), 83-87.

[13] Yi, H.X., Unicity theorems for meromorphic or entire functions II, Bull. Austral. Math. Soc., 53(1995), 71-82.

[14] Yi, H.X., Meromorphic functions that share two sets, (Chinese), Acta Math. Sinica, 45(2002), no. 1, 75-82.

Arindam Sarkar

Department of Mathematics,

Krishnagar Women's College,

Krishnagar, West Bengal, India-741101

e-mail: sarkararindam.as@gmail.com 